\title{
Temperature measurements in a laminar plasma jet generated at reduced pressure
}

\author{
Xian Meng ${ }^{\mathrm{a}, *}$, Wenxia Pan ${ }^{\mathrm{a}}, \mathrm{Xi}$ Chen ${ }^{\mathrm{b}}$, Zhiying Guo ${ }^{\mathrm{a}}$, Chengkang $\mathrm{Wu}^{\mathrm{a}}$ \\ ${ }^{a}$ Institute of Mechanics, Chinese Academy of Sciences, Beijing 100190, China \\ ${ }^{\mathrm{b}}$ Department of Engineering Mechanics, Tsinghua University, Beijing 100084, China
}

\section{A R T I C L E I N F O}

Article history:

Received 5 May 2010

Received in revised form

22 October 2010

Accepted 12 November 2010

\section{Keywords:}

Laminar plasma jet

Reduced pressure

Temperatures measurement

Heat transfer

\begin{abstract}
A B S T R A C T
Double electrostatic probe method and Boltzmann-plot method are applied to measure the electron temperature and the excitation temperature of a DC laminar argon plasma jet generated at reduced pressure. Attempts are also made to measure the gas temperature by using a pair of WRe-5/26 thermocouple. Experimental results show that the measured excitation temperature at the jet center increases with the vacuum chamber pressure and the arc current, but is appreciably lower than the measured electron temperature, indicating that the plasma jet under study is evidently deviated from the LTE state. The temperature indicated by the thermocouple probe is much lower than the measured excitation temperature and electron temperature. Theoretical analysis shows that the thermocouple method intended for gas temperature measurement contains very large errors due to rarefied-gas heat transfer effects, and thus is unsuitable for this purpose.
\end{abstract}

(c) 2010 Elsevier Ltd. All rights reserved.

\section{Introduction}

DC arc plasma jet generated at reduced pressure has the features of large plasma volume, moderate energy density and comparatively small temperature and velocity gradients, which are favorable for depositing large area films/coatings [1-3]. However, this kind of surface-processing technology still relies more on operators' experiences in practical use, poor understanding of the basic plasma phenomena has been the major obstacles to further development of the technology. Therefore, it is useful to systematically diagnose the plasma jet parameters, especially the jet temperatures and their distributions, on which the process suitability and control depend, for better understanding and use of this kind of plasma jet.

Generally, thermal plasmas produced in high intensity arcs at atmospheric pressure can be considered to be in local thermodynamic equilibrium (LTE) state, which is characterized by approximately equal heavy-particle and electron temperatures [4]. In the past decades, many papers have been published to report the measured results about the temperatures of turbulent plasma jets issuing from non-transferred arc plasma torches or the temperatures of the transferred arcs operating at

\footnotetext{
* Corresponding author. Fax: +861062561284.

E-mail address: mengxian@imech.ac.cn (X. Meng).
}

atmospheric pressure [5-7]. However, for the plasmas generated at reduced pressure, deviations from the LTE state may occur since lower electron number density and lesser collisions between gas particles are involved. For this case, the plasma is often characterized by means of several different temperatures, such as the electron temperature $T_{e}$, the excitation temperature $T_{e x}$, the translational temperature of heavy particles $T_{c}$, the vibration temperature $T_{v}$, and the rotational temperature $T_{r}$ [4]. Different methods are usually required to measure the different temperatures. However, so far diagnostic research about the parameters of the plasma jets generated at reduced pressure is relatively scarce.

A specially designed non-transferred DC arc argon plasma torch with a large anode-nozzle exit diameter for use in reduced pressure environment has been constructed [8-12] and employed in this study. The electron temperature and the excitation temperature of the DC arc plasma jets generated at reduced pressure are measured by using the double electrostatic-probe method $[13,14]$ and the spectroscopic-intensity Boltzmann-plot method [4] respectively, and the thermocouple method is also used as an attempt to measure the gas temperature. The reason for the significant deviation of the gas temperature indicated by the thermocouple reading from the measured excitation temperature has been analyzed based on a theoretical analysis considering the rarefiedgas effects on heat transfer from the low-pressure plasma flow to the thermocouple. 


\section{Experimental details}

The non-transferred DC arc plasma torch employed for generating the plasma jet at reduced pressures is the same as that used in the former work [8-12], which mainly contains an anode, a cathode and a three-piece inter-electrode insert. The plasma torch is installed on the top of a vacuum chamber, and the plasma jet is issuing freely into the vacuum chamber. The diameter and height of the vacuum chamber are $650 \mathrm{~mm}$ and $450 \mathrm{~mm}$, respectively. Plasma jets are generated using argon as the working gas with flow rate of $1.25 \times 10^{-4} \mathrm{~kg} / \mathrm{s}$, arc current of $80 \mathrm{~A}-110 \mathrm{~A}$ and vacuum chamber pressure of $170 \mathrm{~Pa}-2000 \mathrm{~Pa}$, where the laminar flow regime is achieved. Photos of the laminar plasma jets for different arc currents and chamber pressures [8] show that the volume of the luminous region of the plasma jet does not increase obviously with increasing arc current, but the jet center region looks somewhat brighter at higher arc current. At higher chamber pressure, the size of luminous region of the plasma jet is appreciably reduced but the jet central region becomes much brighter, indicating the notable decrease of the plasma volume and the appreciable increase of the plasma temperature and its radial gradient at higher gas pressure.

The schematic diagram of the experimental setup is shown in Fig. 1. A double electrostatic probe system has been applied to measure the electron temperature $T_{e}$. The probe system mainly consists of five parts, i.e., two fine-wire electrodes for the double electrostatic probe, one boron nitride probe holder, a DC bias power supply, a sampling resistance of $10 \Omega$ and an oscilloscope for collecting the voltage signals across the sampling resistance and the biased power supply. The double electrostatic probe comprises two individual tungsten wire electrodes of $0.3 \mathrm{~mm}$ in diameter with an exposed length of $2 \mathrm{~mm}$ in the plasma flow. Other surface of each tungsten wire is covered by an alumina tube of $0.5 \mathrm{~mm}$ inner diameter, $1 \mathrm{~mm}$ outer diameter and $35 \mathrm{~mm}$ length. The probe head is fixed in the boron nitride holder with a center distance of $2 \mathrm{~mm}$ between the two tungsten-wire electrodes. The holder can move in the axial and radial direction of the plasma jet. According to the theory of the double electrostatic probe $[13,14]$, the electron temperature $T_{e}$ can be deduced from the measured voltage-current
(V-I) characteristic curve and the electron temperature is presented here as the averaged value of at least five measured data at each spatial position.

The spectroscopic measurement system consists of a spectrometer with focal length of $550 \mathrm{~mm}$, an ICCD detector, an external optical imaging and transmission part including a lens with focal length of $300 \mathrm{~mm}$ and an optical fiber of $0.2 \mathrm{~mm}$ in diameter. The image of the plasma jet is formed on the frontal end of the optical fiber through the lens, and then transmitted to the slit of the spectrometer by the optical fiber. The ICCD can detect the light intensities of chosen spectral lines eventually.

The Boltzmann-plot method [4] is used to obtain the excitation temperature $T_{e x}$ of the plasma jet. Six ArI spectral lines $(675.28 \mathrm{~nm}$, $687.13 \mathrm{~nm}, 696.54 \mathrm{~nm}, 706.72 \mathrm{~nm}, 738.40 \mathrm{~nm}$, and $750.39 \mathrm{~nm}$ ) have been employed to draw the Boltzmann-plot line, and the electron temperature is determined from the slope of the fitted straight line. The spectroscopic parameters of the selected ArI spectral lines are taken from the atomic database of NIST [15]. The exposure time for getting the integrated intensities of each ArI spectral line is set to $100 \mathrm{~ms}$ in this experiment.

A thermocouple probe made of $0.2 \mathrm{~mm}$ diameter WRe-5/26 wires is used in an attempt to measure the gas temperature. A twohole ceramic tube with outer diameter of $4.1 \mathrm{~mm}$ was used to fix the thermocouple wires and support the thermocouple. The distance between the thermocouple ball-shaped junction and the frontal end of ceramic tube is taken to be $4 \mathrm{~mm}$ in order to reduce the conduction loss along the thermocouple wires. The ceramic tube was tied to a water-cooled holder which can move in the axial and radial direction of the plasma jet. Initially, the thermocouple was located outside the plasma jet, and it is moved to a desired measurement position quickly by means of the translation mechanism for measuring the local gas temperature. The temperature indicated by the thermocouple probe is denoted as $T_{p}$ in this paper. A data-collecting system with sampling rate of $1 \mathrm{kHz}$ was used to record the temperature evolution history from the value before putting the thermocouple into the plasma jet to the equilibrium temperature finally reached at the measuring position. Experimental results show that due to the existence of the thermal inertia

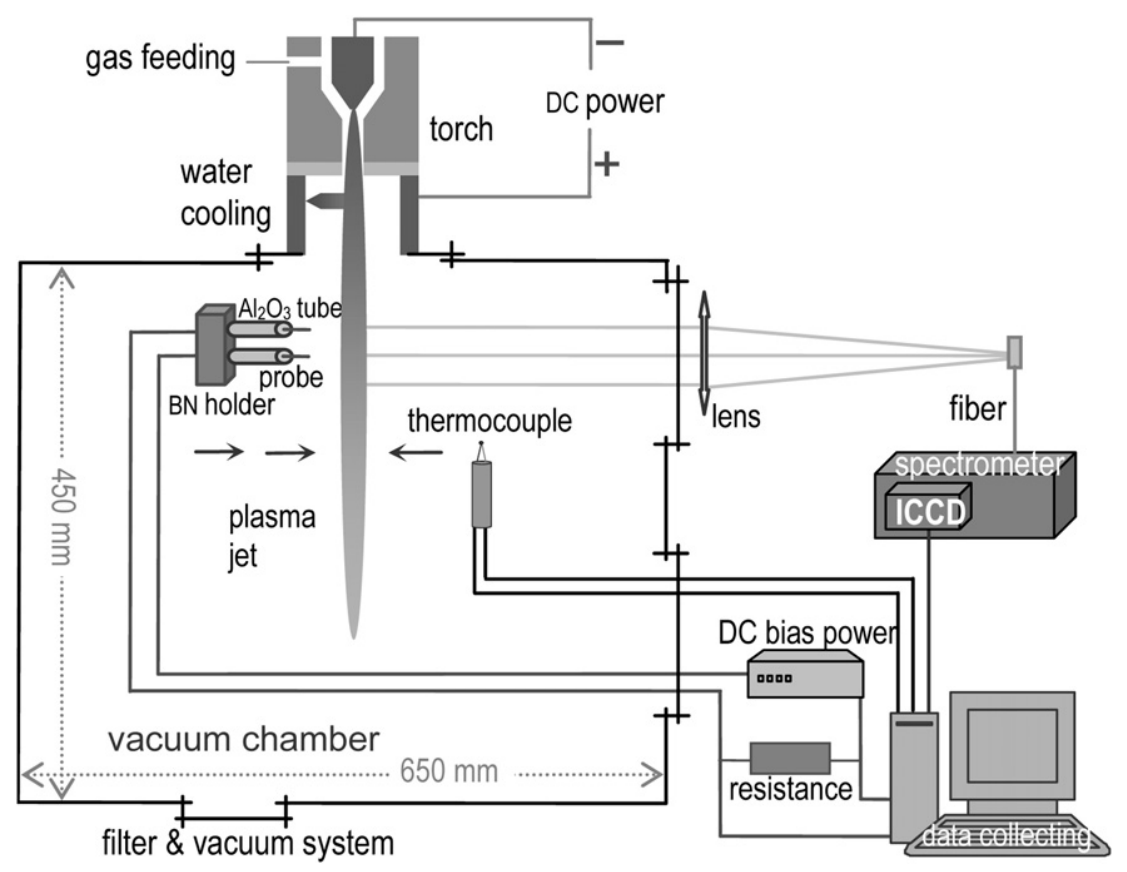

Fig. 1. Schematic diagram of the experimental setup. 


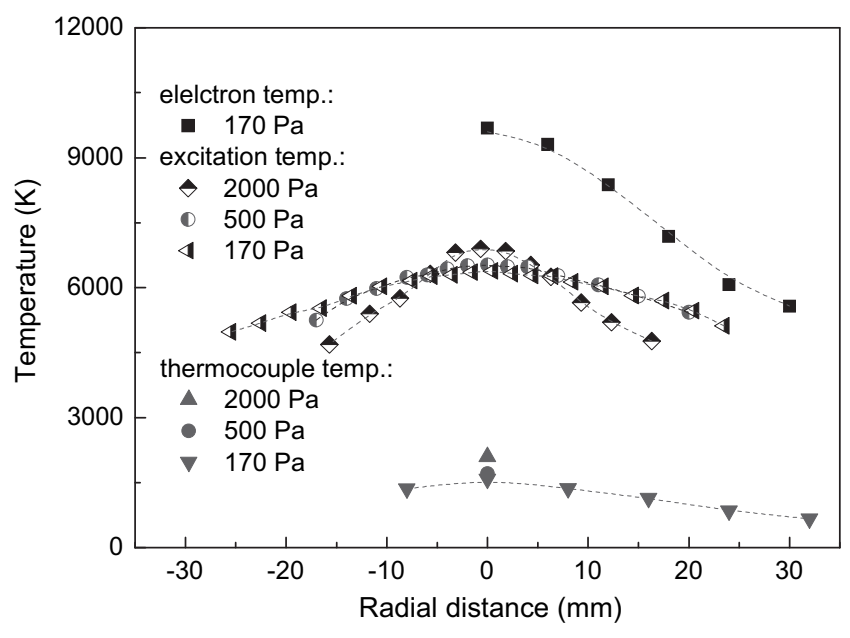

Fig. 2. Radial distributions of the measured electron temperature, excitation temperature and gas temperature of the laminar argon plasma jet for the case with argon flow-rate of $1.25 \times 10^{-4} \mathrm{~kg} / \mathrm{s}$, arc current of $80 \mathrm{~A}$ and axial distance of $10 \mathrm{~mm}$.

of thermocouple probe, it takes about $4.5 \mathrm{~s}$ for the thermocouple probe to achieve the equilibrium temperature, i.e., achieve such a state where the heat flux due to the convective heat transfer from the plasma jet to the ball-shaped junction of the thermocouple is equal to the radiation heat loss from the thermocouple junction to the cold surrounding plus the conductive heat loss (if any) from the thermocouple junction to the ceramic tube along the thermocouple wires. Hence, in this study, the thermocouple stay time at each spatial position is always adopted to be at least $5 \mathrm{~s}$ to get the steady temperature $T_{p}$.

\section{Results and discussions}

\subsection{Measured electron temperature, excitation temperature and gas temperature}

Fig. 2 plots the measured radial distributions of the electron temperature $T_{e}$, the excitation temperature $T_{e x}$ and the gas temperature $T_{p}$ of the argon plasma jet for the case with a fixed axial distance of $10 \mathrm{~mm}$ and a constant arc current of $80 \mathrm{~A}$. For the $T_{e}$ measurements, the vacuum chamber pressure is fixed at $170 \mathrm{~Pa}$, while the chamber pressure varies from $170 \mathrm{~Pa}$ to $2000 \mathrm{~Pa}$ for the $T_{e x}$ and $T_{p}$ measurements. It is clearly seen that the values of $T_{e}$ are much greater than those of $T_{e x}$, while the values of $T_{p}$ are much lower than those of $T_{e}$ and $T_{e x}$. The electron temperature $T_{e}$ is as high as $9600 \mathrm{~K}$ at the jet center, while it decreases to about $5600 \mathrm{~K}$ at the radial position of $30 \mathrm{~mm}$, i.e. the mean radial temperature gradient is about $130 \mathrm{~K} / \mathrm{mm}$. The excitation temperature $T_{e x}$ at the jet center is about $6400 \mathrm{~K}$ for the chamber pressure of $170 \mathrm{~Pa}$, and its mean radial gradient is about $55 \mathrm{~K} / \mathrm{mm}$, which is appreciably smaller than that of $T_{e}$. When the chamber pressure increases to $2000 \mathrm{~Pa}$, the excitation temperature at the jet center increases to about $6900 \mathrm{~K}$. It is also clearly seen that with the increase of the chamber pressure, the mean radial gradient of $T_{e x}$ increases. It increases from about $55 \mathrm{~K} / \mathrm{mm}$ at the chamber pressure of 170 Pa to about $65 \mathrm{~K} /$ $\mathrm{mm}$ at $500 \mathrm{~Pa}$, and then to $130 \mathrm{~K} / \mathrm{mm}$ at $2000 \mathrm{~Pa}$. The temperature measured by the thermocouple is about $1600 \mathrm{~K}$ at the jet center and decreases to $620 \mathrm{~K}$ at the jet edge when the chamber pressure is $170 \mathrm{~Pa}$, and the radial gas temperature gradient is about $30 \mathrm{~K} / \mathrm{mm}$.

Fig. 3 provides the measured results about the distributions of $T_{e}$, $T_{e x}$ and $T_{p}$ along the jet axis for the case with a fixed chamber pressure of $170 \mathrm{~Pa}$ and a constant arc current of $80 \mathrm{~A}$. It is seen that

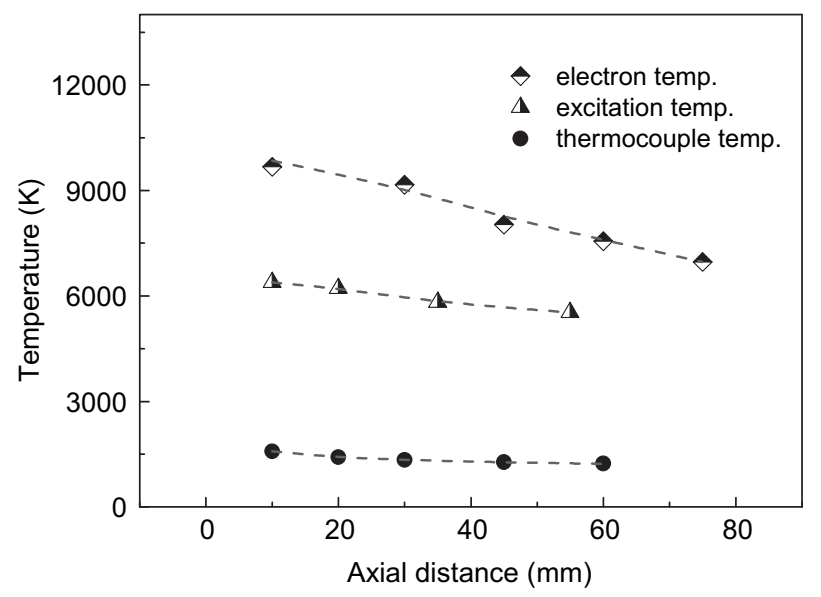

Fig. 3. Axial distributions of the electron temperature, excitation temperature and gas temperature measured at the jet axis for the case with argon flow-rate of $1.25 \times 10^{-4} \mathrm{~kg} / \mathrm{s}$, arc current of $80 \mathrm{~A}$ and chamber pressure of $170 \mathrm{~Pa}$.

all the three kinds of temperatures decrease with increasing axial distance between the torch exit and the measured position. For the electron temperature $T_{e}$, it decreased from $9600 \mathrm{~K}$ at the axial distance of $10 \mathrm{~mm}$ to about $7000 \mathrm{~K}$ at the axial distance of $75 \mathrm{~mm}$. The mean axial gradient of the electron temperature is about $40 \mathrm{~K}$ / $\mathrm{mm}$, which is much smaller than its mean radial gradient $130 \mathrm{~K} / \mathrm{mm}$. The mean axial gradient of the excitation temperature $T_{e x}$ is about $20 \mathrm{~K} / \mathrm{mm}$ and smaller than its mean radial gradient of $55 \mathrm{~K} / \mathrm{mm}$. The averaged axial gradient of the gas temperature $T_{p}$ is about $6.5 \mathrm{~K} / \mathrm{mm}$, which is also much smaller than its radial gradient $30 \mathrm{~K} / \mathrm{mm}$. The smaller axial gradients of temperatures $T_{e}, T_{e x}$ and $T_{p}$ indicate that the laminar plasma jet may preserve its state of high temperature along the jet axis for a large distance.

The variations of $T_{e}, T_{e x}$ and $T_{p}$ with the arc current are shown in Fig. 4 for the case with a fixed chamber pressure of $170 \mathrm{~Pa}$. It is clearly seen that the electron temperature $T_{e}$ increases with increasing arc current. When the arc current is increased from $80 \mathrm{~A}$ to $100 \mathrm{~A}$, the electron temperature increases from $9600 \mathrm{~K}$ to $13000 \mathrm{~K}$. The variations with the arc current of the excitation temperature $T_{e x}$ and the gas temperature $T_{p}$ are not as appreciable as the electron temperature $T_{e}$, but they also increase with the arc current. With the increase of the arc current, the input power and

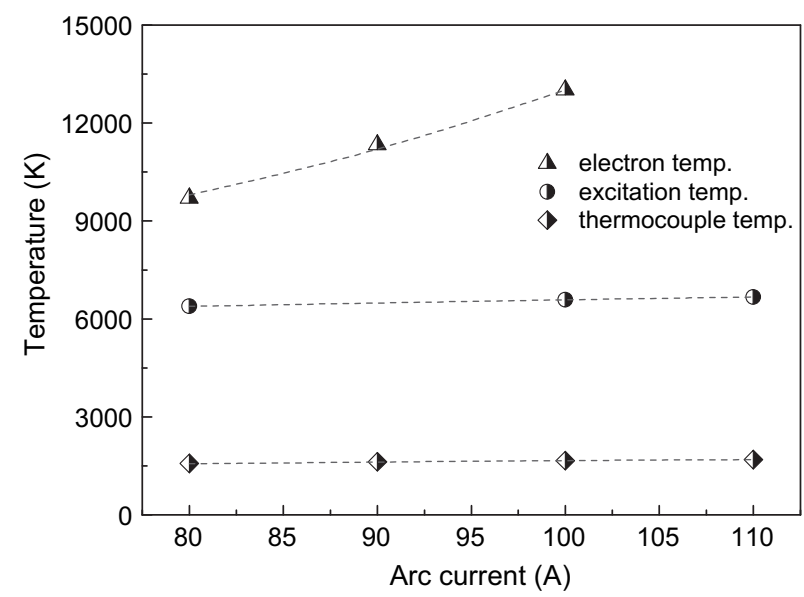

Fig. 4. Variations with the arc current of the measured electron temperature, excitation temperature and gas temperature for the case with argon flow-rate of $1.25 \times 10^{-4} \mathrm{~kg} / \mathrm{s}$, chamber pressure of $170 \mathrm{~Pa}$ and axial distance of $10 \mathrm{~mm}$. 
thus the jet temperature increase, which is consistent with the observation from the photos of the laminar plasma jet [8] mentioned above.

From the measured results presented in Figs. 2-4, it is clear that the electron temperature $T_{e}$ is significantly higher than the excitation temperature $T_{e x}$ and very much higher than the measured thermocouple temperature $T_{p}$ under the same working conditions. The plasma jet generated at reduced pressure is evidently deviated from the local thermodynamic equilibrium state, which is different from the arc plasma jet generated at atmospheric pressure.

\subsection{Discussion on the measuring error of the gas temperature using the thermocouple}

The measured results show that the temperature $T_{p}$ is much lower than the excitation temperature $T_{e x}$. It is believed that such a significant difference between $T_{e x}$ and $T_{p}$ is mainly due to the fact that large systematic error exists in attempting to measure the gas temperature by the thermocouple method under the present conditions, as shown by the following analysis.

Suppose a typical value of the measured temperatures $T_{p}$ presented in Figs. $2-4$ is taken to be $1600 \mathrm{~K}$ at the jet center for the case with the argon mass flow rate of $1.25 \times 10^{-4} \mathrm{~kg} / \mathrm{s}$, arc current of $80 \mathrm{~A}$ and chamber pressure of $170 \mathrm{~Pa}$, and assume that this temperature were the true gas temperature. From the well-known equation for the sound speed of prefect gas $a_{s}=(\gamma R T)^{1 / 2}$ (where $T, R$ and $\gamma$ are the gas temperature, ideal gas constant and specific heat ratio, whereas $a_{s}$ is the sound speed), the sound speed corresponding to $1600 \mathrm{~K}$ argon would be about $742 \mathrm{~m} / \mathrm{s}$. Former velocity measurement by means of an electrostatic probe method [16] of the laminar plasma jet issuing from the same plasma torch under the same working conditions (argon mass flow rate of $1.25 \times 10^{-4} \mathrm{~kg} / \mathrm{s}$, arc current of $80 \mathrm{~A}$ and chamber pressure of $170 \mathrm{~Pa}$ ) showed that the measured mean velocity at the jet center was about $1200 \mathrm{~m} / \mathrm{s}$. Hence, if the gas temperature were indeed $1600 \mathrm{~K}$, the Mach number (=velocity/sound speed) at the center of the measuring section would have been about 1.6, i.e. the flow of the laminar plasma jet at the measuring position would have been supersonic for this case. However, when we inserted a tungsten stick of $1.5 \mathrm{~mm}$ diameter into this laminar plasma jet, there was not any indication of occurrence of Mach waves, as demonstrated in Fig. 5(a). As a comparison, Fig. 5(b) shows a photo which demonstrates that when a tungsten stick is inserted into a truly supersonic plasma flow, Mach waves appear around the tungsten stick, as expected. This fact tells us that the laminar plasma jet shown in

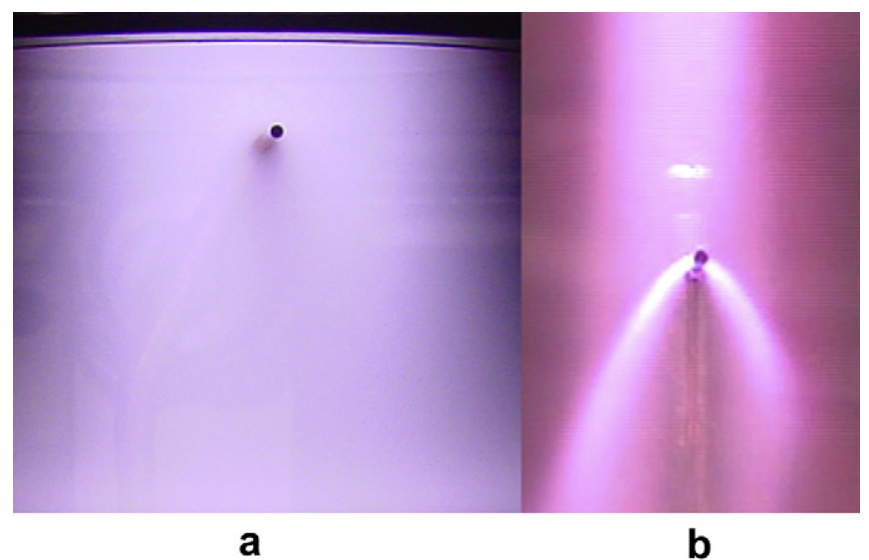

Fig. 5. Photographs obtained when a tungsten stick is inserted into (a) the laminar argon plasma jet at chamber pressure of $170 \mathrm{~Pa}$ and (b) a supersonic plasma jet.
Fig. 5(a) must not have been supersonic, i.e. the local sound speed must have been much higher than $742 \mathrm{~m} / \mathrm{s}$ and the actual gas temperature must have been much higher than the $1600 \mathrm{~K}$ measured by the thermocouple. Since the local sound speed must have been higher than the gas flow velocity $1200 \mathrm{~m} / \mathrm{s}$, correspondingly the gas temperature must have been at least higher than about $4200 \mathrm{~K}$, i.e. the gas temperature measured directly by the thermocouple must have contained large error for the present reduced pressure case.

A most likely reason for the existence of such a large error in the measured gas temperature by thermocouple for the present reduced pressure case is that the convective heat transfer from the flowing gas to the thermocouple is reduced significantly at low gas pressure. It is well known that the indicated temperature of the thermocouple is the result of heat balance at the thermocouple surface involving several different heat transfer mechanisms. If we ignore the conductive heat transfer along the thermocouple wires, the heat flux due to the convective heat transfer from the flowing plasma to the thermocouple would be equal to that due to the radiative heat loss from the thermocouple to the cold environment at steady state. The heat flux due to the radiation heat loss from the thermocouple surface $q_{r}$ (in $\mathrm{W} / \mathrm{m}^{2}$ ) can be calculated by $q_{r}=\varepsilon \sigma T_{p}^{4}$, where $\varepsilon$ is the emissivity of the thermocouple material and $\sigma$ is the Stefan-Boltzmann constant. On the other hand, when the gas flow temperature is about $5000 \mathrm{~K}$ and the gas pressure is $170 \mathrm{~Pa}$, the Knudsen number $(\mathrm{Kn})$ would be larger than 1 and thus the convective heat transfer from the flowing gas to the thermocouple junction should be treated as a rarefied-gas heat transfer problem. For the free-molecule regime, Ref. [17] derived the expressions for the heat fluxes to a cylindrical wire due to atoms, ions and electrons, respectively. For the case studied here, degree of ionization of the gas is small and thus the heat transfer will be dominated by argon atoms. Hence, the convective heat flux from the flowing gas to the cylindrical wire surface $q_{c}\left(\right.$ in $\mathrm{W} / \mathrm{m}^{2}$ ) can be calculated by [17]:

$$
\begin{aligned}
q_{c}= & a p_{\infty} \sqrt{\frac{k T_{\infty}}{2 \pi m}} e^{-S^{2} / 2}\left\{\left(2+S^{2}-2 \frac{T_{w}}{T_{\infty}}\right) I_{0}\left(\frac{S^{2}}{2}\right)\right. \\
& \left.+\left(\frac{5}{2}+S^{2}-2 \frac{T_{w}}{T_{\infty}}\right) S^{2}\left[I_{0}\left(\frac{S^{2}}{2}\right)+I_{1}\left(\frac{S^{2}}{2}\right)\right]\right\}
\end{aligned}
$$

where $a$ is the thermal accommodation coefficient at the thermocouple surface, $p_{\infty}, T_{\infty}$, and $U_{\infty}$ are the static pressure, temperature and velocity of the flowing gas, $k$ is the Boltzmann constant, $m$ is the argon atom mass, $T_{w}$ is the wire surface temperature (here $T_{w}=T_{p}$ ), $S=U_{\infty} /\left(2 k T_{\infty} / m\right)^{1 / 2}$ is the so-called speed ratio, whereas $I_{0}(\mathrm{z})$ and $I_{1}(\mathrm{z})$ are the zero-order and first-order modified Bessel function, respectively.

When the heat transfer processes reach the steady state, the convective heat flux $q_{c}$ is equal to the radiative heat flux $q_{r}$, and thus the temperature of the oncoming gas flow can be deduced from the measured temperature $T_{p}$. For the typical case with measured temperature $T_{p}=1600 \mathrm{~K}$ and gas flow velocity $U_{\infty}=1200 \mathrm{~m} / \mathrm{s}$, Fig. 6 shows the variations with gas-flow temperature of the convective and radiative heat fluxes using the thermal accommodation factor $a$ and the material emissivity $\varepsilon$ as two parameters. One can see from Fig. 6 that reliable data of the emissivity of the thermocouple material and the thermal accommodation coefficient at the thermocouple surface are required for accurately deducing the gas flow temperature from $q_{c}=q_{r}$. The deduced gas temperature will decrease with decreasing emissivity $\varepsilon$ and increase with decreasing thermal accommodation coefficient $a$. If the thermal accommodation coefficient $a$ of the WRe-5/26 thermocouple surface is taken to be 1.0 and the tungsten emissivity $\varepsilon$ is taken to be 


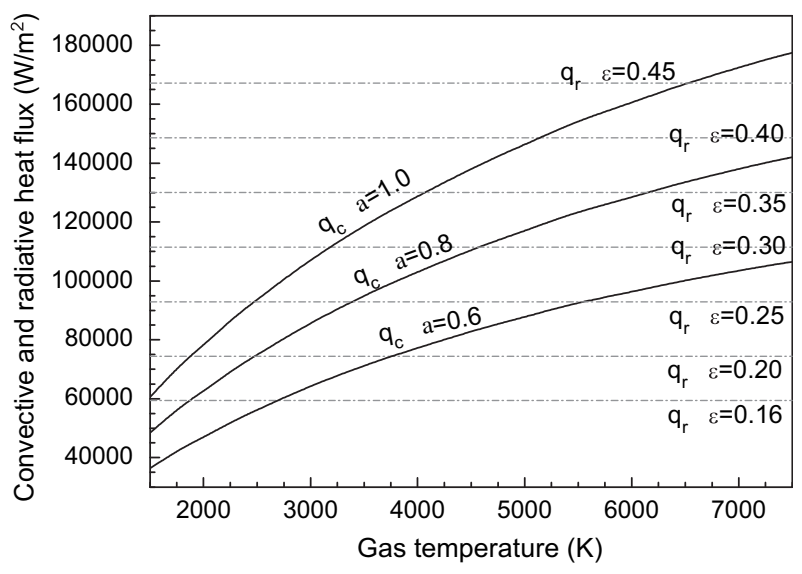

Fig. 6. Variations of the convective and radiative heat flux with gas temperature $T_{\infty} . T_{p}$, $u_{\infty}$ and $p_{\infty}$ are taken to be $1600 \mathrm{~K}, 1200 \mathrm{~m} / \mathrm{s}$ and $170 \mathrm{~Pa}$, respectively, in the calculation.

0.4 [18], the gas temperature $T_{\infty}$ deduced from Fig. 10 is $5100 \mathrm{~K}$. On the other hand, when $a=0.8$ and $\varepsilon=0.35$ are adopted, the deduced gas temperature $T_{\infty}$ would be $6100 \mathrm{~K}$. Although the uncertainty in $\varepsilon$ and/or $a$ influences the value of the deduced gas temperature, the deduced gas temperature $T_{\infty}$ is always much higher than the temperature $T_{p}$ measured by the thermocouple. It means that the temperature measurement using the WRe-5/26 thermocouple at low gas pressure always contains large measuring errors, and the $T_{p}$ value given by the thermocouple cannot be taken directly as the gas temperature.

\section{Conclusions}

In this study, the electron temperature and the excitation temperature of the non-transferred DC argon plasma jet generated at reduced pressure are measured by using double electrostatic probe method and the spectroscopic-intensity Boltzmann-plot method, respectively. Attempts are also made to measure the gas temperature by using a pair of WRe-5/26 thermocouple. Experimental results show that the electron temperature is as high as $9600 \mathrm{~K}$ for the case with gas flow rate of $1.25 \times 10^{-4} \mathrm{~kg} / \mathrm{s}$, arc current of $80 \mathrm{~A}$ and vacuum chamber pressure of $170 \mathrm{~Pa}$, while the excitation temperature and the thermocouple temperature are about $6400 \mathrm{~K}$ and $1600 \mathrm{~K}$ respectively at the same working conditions. These three kinds of temperatures all increase with increasing arc current and decrease with increasing axial distance. The measured electron temperature is much higher than corresponding excitation temperature, indicating that the low-pressure plasma jet is evidently deviated from the local thermodynamic equilibrium state under the present experimental conditions. Theoretical analysis based on a rarefied-gas heat transfer approach shows that temperatures of the WRe-5/26 thermocouple probe would have values very much below the gas stream temperature under these conditions, and thus cannot represent actual temperature of the gas.

\section{Acknowledgements}

This work is supported by the National Natural Science Foundation of China (Nos. 50836007, 10805066 and 10775164).

\section{References}

[1] Pan WX, Lv FX, Tang WZ, Zhong GF, Jiang Z, Wu CK. Diamond and Related Materials 2000;9(9-10):1682.

[2] Marcinauskas L, Grigonis A, Kulikauskas V, Valincius V. Vacuum 2007;81 (10): 1220

[3] Kambara M, Hamai Y, Yagi H, Yoshida T, Surface. Coatings Technology 2007;201(9-11):5529.

[4] Boulos M, Fauchais P, Pfender E. Diagnostic techniques in thermal plasma processing, part I. U.S. Department of Energy; 1986.

[5] Semenov S, Cetegen B. Journal of Thermal Spray Technology 2001;10:326.

[6] Zhao WH, Li JQ, Yan JD. IEEE Transactions on Plasma Science 1997;25:828.

[7] Pfender E, Spores R, Chen WLT. International Journal of Material Product Technology 1995;10:548.

[8] Pan WX, Guo ZY, Meng X, Huang HJ, Wu CK. Plasma Sources Science and Technology 2009;18:045032.

[9] Pan WX, Li T, Meng X, Chen X, Wu CK. Chinese Physics Letters 2005;22:2895.

[10] Pan WX, Meng X, Li T, Chen X, Wu CK. Plasma Science and Technology 2007;9:152.

[11] Huang HJ, Pan WX, Guo ZY, Wu CK. IEEE Transactions on Plasma Science 2008;36:1052.

[12] Huang HJ, Pan WX, Wu CK. Chinese Physics Letters 2008:25:4058.

[13] Swift JD, Schwar MJR. Electrical probes for plasma diagnostics. London: Iliffe Books Ltd.; 1970.

[14] Henderson S, Menart J, Shang J, Kimmel R, Hayes J. 42nd AIAA aerospace sciences meeting and exhibit. AIAA; 2004. 2004-2360.

[15] NIST Atomic Spectra Database Data, http://physics.nist.gov.

[16] Pan WX, Guo ZY, Meng X, Huang HJ, Wu CK, Proceedings of the 2nd International workshop on non-equilibrium flows, Lijiang, 2008; 1.

[17] Chen X. Journal of Physics D: Applied Physics 1997;30:1885.

[18] Larrabee RD. The spectral emissivity and optical properties of tungsten. Technical Report 328 in MIT; 1957. 